\title{
Peribulbar versus general anesthesia for horizontal strabismus surgery
}

\author{
Anestesia peribulbar versus anestesia geral na cirurgia de estrabismo horizontal
}

\author{
Keila Monteiro de Carvalho ${ }^{1}$ \\ Tatiana Millán² \\ Nilza Minguini ${ }^{3}$ \\ Tais Hitomi Wakamatsu ${ }^{4}$
}

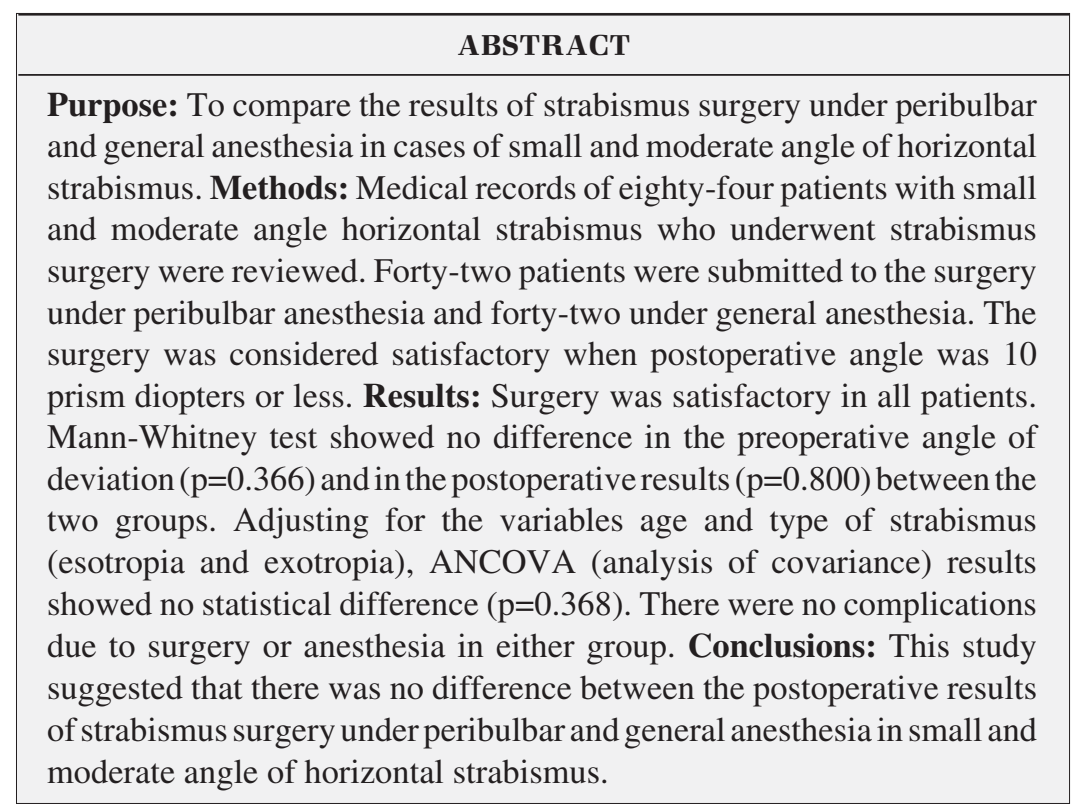

Keywords: Anesthesia, local/methods; Strabismus/surgery; Ophthalmologic surgical procedures; Ocular motility disorders

\section{INTRODUCTION}

Trabalho realizado no Departamento de Oftalmologia/ Otorrinolaringologia da Faculdade de Ciências Médicas da Universidade Estadual de Campinas - UNICAMP - Campinas (SP) - Brasil.

Doutora, Professora e Chefe da Disciplina de Oftalmologia do Departamento de Oftalmologia/Otorrinolaringologia da Faculdade de Ciências Médicas da Universidade Estadual de Campinas - UNICAMP - Campinas (SP) - Brasil.

Mestre, Médica Oftalmologista da Faculdade de Ciências Médicas da UNICAMP - Campinas (SP) - Brasil.

Mestre, Assistente do Hospital das Clínicas da UNICAMP - Campinas (SP) - Brasil.

${ }^{4}$ Médica Oftalmologista da Faculdade de Ciências Médicas da UNICAMP - Campinas (SP) - Brasil.

Endereço para correspondência: Keila Monteiro Carvalho. Rua Capote Valente, 432 - Conj. 155 - São Paulo (SP) CEP 05409-001

E-mail: keilammc@fcm.unicamp.br

Recebido para publicação em 21.08.2007

Última versão recebida em 09.10.2007

Aprovação em 06.12.2007

Nota Editorial: Depois de concluída a análise do artigo sob sigilo editorial e com a anuência da Dra. Mônica Fialho Cronemberger sobre a divulgação de seu nome como revisora, agradecemos sua participação neste processo.

\footnotetext{
Strabismus surgery is commonly performed under general anesthesia. However, it has been suggested that peribulbar anesthesia may be associated with decreased morbidity in patients undergoing surgery to correct strabismus $\mathrm{s}^{(1-3)}$. In addition, it is a technique with minimal equipment and low cost and causes less nausea/vomiting and recovery is earlier than with general anesthesia ${ }^{(1,4-5)}$. Peribulbar anesthesia can potentially lower the risk of anesthesia in patients with poor general health, cardiac disease, hypertension or history of complications in previous general anesthesia. The disadvantages of this type of anesthesia include less mobility of the globe, which hinders the analysis of passive strengths ${ }^{(6)}$.

In many public health services general anesthesia availability is not sufficient to meet the demand. Therefore and in an attempt to reduce the morbidity and mortality associated with general anesthesia, particularly in those patients with high-risk characteristics, we decided to perform a study to expand the use of peribulbar anesthesia in strabismus surgery.

This study has the objective of comparing the results of strabismus surgery under peribulbar and general anesthesia in cases of small angle horizontal strabismus.
} 


\section{METHODS}

\section{Subjects}

Medical records of patients with horizontal strabismus of small or moderate angle (defined angle of deviation inferior to 50 prism diopters) ${ }^{(7)}$ who underwent primary strabismus procedures under peribulbar and general anesthesia at the Department of Ophthalmology, State University of Campinas, from January 2002 to December 2003 were retrospectively reviewed.

The subjects were divided into groups 1 and 2. Group 1 was consisted of all patients with horizontal strabismus of small angle of deviation who underwent strabismus surgery under peribulbar anesthesia in this period; group 2, used to compare the results with group 1, was composed of the same number of consecutive patients with horizontal strabismus of small angle who underwent strabismus surgery under general anesthesia in this period.

\section{Procedures}

\section{1) Measurement of angle of deviation}

The angle of strabismus was measured in prism diopters (PD) with the best optic correction, in all positions, for distance and near vision. For patients with good bilateral visual acuity the measurement was made by the prism and cover test using a suitable fixation target. Patients with low vision or profound amblyopia were submitted to Krinsky measurement.

\section{2) Surgical planning and surgery}

The surgical technique used for all patients consisted of recession and resection. All patients were submitted to unilateral surgeries using the same surgical planning for each angle of deviation in both groups (peribulbar and general). All patients were informed and accepted to submit to the surgery. A successful result was defined by a postoperative angle of 10 PD or less.

\section{3) Anesthesia}

Peribulbar anesthesia was performed by the ocular surgeon and all patients received $5 \mathrm{mg}$ diazepam orally thirty minutes before the anesthesia. An intravenous line, oxygen nasal cannula, cardiac monitoring and continuous pulse oximetry were employed. The local anesthetic consisted of $0.5 \%$ bupivacaine without epinephrine and $2 \%$ lidocaine with epinephrine. General anesthesia was performed by the anesthetist.

\section{Follow-up}

Patients were examined at least on the first, seventh and thirtieth day and 3 months after surgery. Some patients were also examined six months after surgery. Measurements of angle of deviation were taken on all visits, but the data considered in this study were from the third postoperative month.

\section{Data analysis}

The methods used for data analysis were Mann-Whitney for comparison of age and preoperative and postoperative angle of deviation among the groups and ANCOVA (analysis covariance) used for considering (or adjusting) covariables (age and type of deviation - esotropia or exotropia). A $P$ value of $<0.05$ was considered statistically significant.

This study was approved by the official Research Ethics Committee.

\section{RESULTS}

Forty-one patients ranging from 15 to 57 years with horizontal strabismus of small and moderate angle underwent strabismus surgery under peribulbar anesthesia (group 1) from January 2002 and December 2004 in this service. Twenty-five patients had esotropia and 16 had exotropia. Age, preoperative and postoperative angles of deviation and surgical planning of these patients are shown in Tables 1 (patients with esotropia) and 2 (patients with exotropia).

Forty-one consecutive patients with horizontal strabismus of small and moderate angle who underwent strabismus surgery under general anesthesia (group 2) were used to compare the results. Age ranged from 10 to 18 years. Thirty-one patients of this group had esotropia and 10 had exotropia. Age, preoperative and postoperative angles of deviation and surgical planning of these patients are shown in Tables 3 (patients with esotropia) and 4 (patients with exotropia).

Surgery was considered satisfactory (postoperative deviation of 10 PD or less) in all patients. Mann-Whitney test showed no difference in the preoperative angle of deviation $(\mathrm{p}=0.366)$ and in the postoperative results $(\mathrm{p}=0.800)$ between the two groups, not considering type of strabismus (esotropia or exotropia). Group 2 was younger $(\mathrm{p}<0.001)$. ANCOVA was used for adjusting the variables age and type of strabismus in the comparison of the postoperative results and showed no statistical difference $(\mathrm{p}=0.368)$.

There were no complications from surgery or anesthesia in either group.

\section{DISCUSSION}

When choosing the method of anesthesia, it is very important to consider the effectiveness and safety of the method. Ocular complications of peribulbar anesthesia are rare and include globe perforation, retrobulbar hemorrhage, ptosis, optic nerve injury and central retinal artery occlusion ${ }^{(4-6)}$. Sanders et al. evaluated the effectiveness of peribulbar anesthesia in strabismus surgery in a prospective study with 76 patients and suggested that this method of anesthesia is safe with minimal associated morbidity ${ }^{(1)}$. In another study, Ripart et al. compared peribulbar and retrobulbar techniques and showed that their effectiveness is similar, but the peribulbar 


\begin{tabular}{|c|c|c|c|c|}
\hline Patient № & Age (vears) & Preoperative deviation & Suraical planning & Postonerative deviation (3 months) \\
\hline 1 & 18 & 25 PD & $\mathrm{RC} 5 \mathrm{~mm} \mathrm{MR}+\mathrm{RS} 5 \mathrm{~mm} \mathrm{LR}$ & $5 \mathrm{PD}$ \\
\hline 2 & 21 & 25 PD & $\mathrm{RC} 5 \mathrm{~mm} \mathrm{MR}+\mathrm{RS} 5 \mathrm{~mm} \mathrm{LR}$ & $0^{*}$ \\
\hline 3 & 15 & $30 \mathrm{PD}$ & $\mathrm{RC} 5 \mathrm{~mm} \mathrm{MR}+\mathrm{RS} 6 \mathrm{~mm} \mathrm{LR}$ & 5 PD \\
\hline 4 & 57 & $30 \mathrm{PD}$ & $\mathrm{RC} 5 \mathrm{~mm} \mathrm{MR}+\mathrm{RS} 6 \mathrm{~mm} \mathrm{LR}$ & $6 \mathrm{PD}$ \\
\hline 5 & 20 & $30 \mathrm{PD}$ & $\mathrm{RC} 5 \mathrm{~mm} \mathrm{MR}+\mathrm{RS} 6 \mathrm{~mm}$ LR & $8 \mathrm{PD}$ \\
\hline 6 & 33 & $30 \mathrm{PD}$ & $\mathrm{RC} 5 \mathrm{~mm} \mathrm{MR}+\mathrm{RS} 6 \mathrm{~mm}$ LR & $8 \mathrm{PD}$ \\
\hline 7 & 21 & $30 \mathrm{PD}$ & $\mathrm{RC} 5 \mathrm{~mm} \mathrm{MR}+\mathrm{RS} 6 \mathrm{~mm}$ LR & $0^{*}$ \\
\hline 8 & 28 & $35 \mathrm{PD}$ & $\mathrm{RC} 5 \mathrm{~mm} \mathrm{MR}+\mathrm{RS} 7 \mathrm{~mm}$ LR & $0^{*}$ \\
\hline 9 & 18 & $35 \mathrm{PD}$ & $\mathrm{RC} 5 \mathrm{~mm} \mathrm{MR} \mathrm{+RS} 7 \mathrm{~mm}$ LR & $5 \mathrm{PD}$ \\
\hline 10 & 19 & $35 \mathrm{PD}$ & $\mathrm{RC} 5 \mathrm{~mm} \mathrm{MR}+\mathrm{RS} 7 \mathrm{~mm}$ LR & $0^{*}$ \\
\hline 11 & 29 & 35 PD & $\mathrm{RC} 5 \mathrm{~mm} \mathrm{MR} \mathrm{+RS} 7 \mathrm{~mm}$ LR & 6 PD \\
\hline 12 & 53 & 35 PD & $\mathrm{RC} 5 \mathrm{~mm} \mathrm{MR}+\mathrm{RS} 7 \mathrm{~mm}$ LR & $0^{*}$ \\
\hline 13 & 53 & 35 PD & $\mathrm{RC} 5 \mathrm{~mm} \mathrm{MR}+\mathrm{RS} 7 \mathrm{~mm}$ LR & $8 \mathrm{PD}$ \\
\hline 14 & 27 & $40 \mathrm{PD}$ & $\mathrm{RC} 5 \mathrm{~mm} \mathrm{MR}+\mathrm{RS} 8 \mathrm{~mm}$ LR & $10 \mathrm{PD}$ \\
\hline 15 & 24 & $40 \mathrm{PD}$ & $\mathrm{RC} 5 \mathrm{~mm} \mathrm{MR}+\mathrm{RS} 8 \mathrm{~mm} \mathrm{LR}$ & $8 \mathrm{PD}$ \\
\hline 16 & 21 & 40 PD & $\mathrm{RC} 5 \mathrm{~mm} \mathrm{MR}+\mathrm{RS} 8 \mathrm{~mm} \mathrm{LR}$ & $6 \mathrm{PD}$ \\
\hline 17 & 24 & $40 \mathrm{PD}$ & $\mathrm{RC} 5 \mathrm{~mm} \mathrm{MR}+\mathrm{RS} 8 \mathrm{~mm} \mathrm{LR}$ & $8 \mathrm{PD}$ \\
\hline 18 & 57 & $40 \mathrm{PD}$ & $\mathrm{RC} 6 \mathrm{~mm} \mathrm{MR}+\mathrm{RS} 7 \mathrm{~mm}$ LR & 6 PD \\
\hline 19 & 15 & 40 PD & $\mathrm{RC} 5 \mathrm{~mm} \mathrm{MR} \mathrm{+RS} 8 \mathrm{~mm}$ LR & 8 PD \\
\hline 20 & 21 & 45 PD & $\mathrm{RC} 5 \mathrm{~mm} \mathrm{MR}+\mathrm{RS} 8 \mathrm{~mm}$ LR & 4 PD \\
\hline 21 & 20 & 45 PD & $\mathrm{RC} 6 \mathrm{~mm} \mathrm{MR}+\mathrm{RS} 8 \mathrm{~mm} \mathrm{LR}$ & $10 \mathrm{PD}$ \\
\hline 22 & 22 & $45 \mathrm{PD}$ & $\mathrm{RC} 6 \mathrm{~mm} \mathrm{MR}+\mathrm{RS} 8 \mathrm{~mm} \mathrm{LR}$ & $10 \mathrm{PD}$ \\
\hline 23 & 20 & $45 \mathrm{PD}$ & $\mathrm{RC} 5 \mathrm{~mm} \mathrm{MR}+\mathrm{RS} 8 \mathrm{~mm}$ LR & $8 \mathrm{PD}$ \\
\hline 24 & 17 & $45 \mathrm{PD}$ & $\mathrm{RC} 5 \mathrm{~mm} \mathrm{MR}+\mathrm{RS} 8 \mathrm{~mm}$ LR & $0^{*}$ \\
\hline 25 & 22 & 45 PD & $\mathrm{RC} 6 \mathrm{~mm} \mathrm{MR}+\mathrm{RS} 7 \mathrm{~mm} \mathrm{LR}$ & $10 \mathrm{PD}$ \\
\hline Mean & 27 & 36.8 PD & & 5.56 PD \\
\hline
\end{tabular}

\begin{tabular}{|c|c|c|c|c|}
\hline Patient № & Age (years) & Preoperative deviation & Surgical planning & Postoperative deviation (3 months) \\
\hline 1 & 37 & $25 \mathrm{PD}$ & $\mathrm{RC} 6 \mathrm{~mm} \mathrm{LR}+\mathrm{RS} 5 \mathrm{~mm} \mathrm{MR}$ & $4 \mathrm{PD}$ \\
\hline 2 & 27 & $30 \mathrm{PD}$ & $\mathrm{RC} 6 \mathrm{~mm} \mathrm{LR}+\mathrm{RS} 5 \mathrm{~mm} \mathrm{MR}$ & $0^{*}$ \\
\hline 3 & 35 & $35 \mathrm{PD}$ & $\mathrm{RC} 6 \mathrm{~mm} \mathrm{LR}+\mathrm{RS} 5 \mathrm{~mm} \mathrm{MR}$ & $4 \mathrm{PD}$ \\
\hline 4 & 29 & $35 \mathrm{PD}$ & $\mathrm{RC} 6 \mathrm{~mm} \mathrm{LR}+\mathrm{RS} 5 \mathrm{~mm} \mathrm{MR}$ & $2 \mathrm{PD}$ \\
\hline 5 & 35 & $35 \mathrm{PD}$ & $\mathrm{RC} 6 \mathrm{~mm} \mathrm{LR}+\mathrm{RS} 5 \mathrm{~mm} \mathrm{MR}$ & $6 \mathrm{PD}$ \\
\hline 6 & 23 & $40 \mathrm{PD}$ & $\mathrm{RC} 8 \mathrm{~mm} \mathrm{LR}+\mathrm{RS} 7 \mathrm{~mm}$ MR & $10 \mathrm{PD}$ \\
\hline 7 & 21 & $40 \mathrm{PD}$ & $\mathrm{RC} 8 \mathrm{~mm} \mathrm{LR}+\mathrm{RS} 7 \mathrm{~mm} \mathrm{MR}$ & $8 \mathrm{PD}$ \\
\hline 8 & 48 & $40 \mathrm{PD}$ & $\mathrm{RC} 8 \mathrm{~mm} \mathrm{LR}+\mathrm{RS} 7 \mathrm{~mm} \mathrm{MR}$ & $10 \mathrm{PD}$ \\
\hline 9 & 31 & $40 \mathrm{PD}$ & $\mathrm{RC} 8 \mathrm{~mm} \mathrm{LR}+\mathrm{RS} 7 \mathrm{~mm} \mathrm{MR}$ & $10 \mathrm{PD}$ \\
\hline 10 & 26 & $40 \mathrm{PD}$ & $\mathrm{RC} 8 \mathrm{~mm} \mathrm{LR}+\mathrm{RS} 7 \mathrm{~mm}$ MR & $6 \mathrm{PD}$ \\
\hline 11 & 38 & $40 \mathrm{PD}$ & $\mathrm{RC} 8 \mathrm{~mm} \mathrm{LR}+\mathrm{RS} 7 \mathrm{~mm}$ MR & 8 PD \\
\hline 12 & 23 & $40 \mathrm{PD}$ & $\mathrm{RC} 7 \mathrm{~mm}$ LR $+\mathrm{RS} 7 \mathrm{~mm} \mathrm{MR}$ & $2 \mathrm{PD}$ \\
\hline 13 & 40 & $45 \mathrm{PD}$ & $\mathrm{RC} 8 \mathrm{~mm} \mathrm{LR}+\mathrm{RS} 7 \mathrm{~mm} \mathrm{MR}$ & $4 \mathrm{PD}$ \\
\hline 14 & 29 & $45 \mathrm{PD}$ & $\mathrm{RC} 8 \mathrm{~mm} \mathrm{LR}+\mathrm{RS} 7 \mathrm{~mm}$ MR & $8 \mathrm{PD}$ \\
\hline 15 & 28 & $45 \mathrm{PD}$ & $\mathrm{RC} 8 \mathrm{~mm} \mathrm{LR}+\mathrm{RS} 7 \mathrm{~mm} \mathrm{MR}$ & $0^{*}$ \\
\hline 16 & 21 & $45 \mathrm{PD}$ & $\mathrm{RC} 8 \mathrm{~mm} \mathrm{LR}+\mathrm{RS} 7 \mathrm{~mm} \mathrm{MR}$ & 8 PD \\
\hline Mean & 30.69 & 38.75 PD & & 5.62 PD \\
\hline
\end{tabular}

technique has fewer risks of ocular complications ${ }^{(8)}$. The shorter needle used in peribulbar anesthesia diminishes the risk of global perforation, in comparison with retrobulbar anesthesia. This is particularly important in myopic eyes with longer globe. The incidence of global perforation after peribulbar anesthesia is very low and occurred in $0.006 \%$ of 16,224 consecutive peribulbar blocks in a multicenter study ${ }^{(4)}$. The intraocular injection of anesthetics is rare, with few reports in the literature ${ }^{(9)}$. Holekamp et al. reported a case of inadvertent intraocular injection of bupivacaine and mepivacaine that had 


\begin{tabular}{|c|c|c|c|c|}
\hline Patient № & Age (years) & Preoperative deviation & Surgical planning & Postoperative deviation (3 months) \\
\hline 1 & 12 & $20 \mathrm{PD}$ & $\mathrm{RC} 4 \mathrm{~mm} \mathrm{MR}+\mathrm{RS} 5 \mathrm{~mm} \mathrm{LR}$ & $0^{*}$ \\
\hline 2 & 15 & $25 \mathrm{PD}$ & $\mathrm{RC} 5 \mathrm{~mm} \mathrm{MR}+\mathrm{RS} 5 \mathrm{~mm} \mathrm{LR}$ & $0^{*}$ \\
\hline 3 & 16 & $30 \mathrm{PD}$ & $\mathrm{RC} 5 \mathrm{~mm} \mathrm{MR}+\mathrm{RS} 6 \mathrm{~mm} \mathrm{LR}$ & $0^{*}$ \\
\hline 4 & 10 & $30 \mathrm{PD}$ & $\mathrm{RC} 5 \mathrm{~mm} \mathrm{MR}+\mathrm{RS} 6 \mathrm{~mm} \mathrm{LR}$ & $4 \mathrm{PD}$ \\
\hline 5 & 11 & $30 \mathrm{PD}$ & $\mathrm{RC} 5 \mathrm{~mm} \mathrm{MR}+\mathrm{RS} 6 \mathrm{~mm} \mathrm{LR}$ & $4 \mathrm{PD}$ \\
\hline 6 & 12 & $30 \mathrm{PD}$ & $\mathrm{RC} 5 \mathrm{~mm} \mathrm{MR}+\mathrm{RS} 6 \mathrm{~mm} \mathrm{LR}$ & $0^{*}$ \\
\hline 7 & 13 & $30 \mathrm{PD}$ & $\mathrm{RC} 5 \mathrm{~mm} \mathrm{MR} \mathrm{+RS} 6 \mathrm{~mm} \mathrm{LR}$ & $5 \mathrm{PD}$ \\
\hline 8 & 13 & $30 \mathrm{PD}$ & $\mathrm{RC} 5 \mathrm{~mm} \mathrm{MR}+\mathrm{RS} 6 \mathrm{~mm} \mathrm{LR}$ & $-2 P D^{\star \star}$ \\
\hline 9 & 17 & $30 \mathrm{PD}$ & $\mathrm{RC} 5 \mathrm{~mm} \mathrm{MR}+\mathrm{RS} 6 \mathrm{~mm} \mathrm{LR}$ & $0^{*}$ \\
\hline 10 & 10 & $35 \mathrm{PD}$ & $\mathrm{RC} 5 \mathrm{~mm} \mathrm{MR}+\mathrm{RS} 7 \mathrm{~mm} \mathrm{LR}$ & $0^{*}$ \\
\hline 11 & 11 & $35 \mathrm{PD}$ & $\mathrm{RC} 5 \mathrm{~mm} \mathrm{MR}+\mathrm{RS} 7 \mathrm{~mm} \mathrm{LR}$ & $2 \mathrm{PD}$ \\
\hline 12 & 10 & $35 \mathrm{PD}$ & $\mathrm{RC} 5 \mathrm{~mm} \mathrm{MR}+\mathrm{RS} 7 \mathrm{~mm} \mathrm{LR}$ & $0^{*}$ \\
\hline 13 & 12 & $35 \mathrm{PD}$ & $\mathrm{RC} 5 \mathrm{~mm} \mathrm{MR} \mathrm{+RS} 7 \mathrm{~mm} \mathrm{LR}$ & $2 \mathrm{DP}$ \\
\hline 14 & 15 & $35 \mathrm{PD}$ & $\mathrm{RC} 5 \mathrm{~mm} \mathrm{MR}+\mathrm{RS} 7 \mathrm{~mm} \mathrm{LR}$ & $0^{*}$ \\
\hline 15 & 14 & $35 \mathrm{PD}$ & $\mathrm{RC} 5 \mathrm{~mm} \mathrm{MR} \mathrm{+RS} 7 \mathrm{~mm} \mathrm{LR}$ & $2 \mathrm{PD}$ \\
\hline 16 & 16 & $35 \mathrm{PD}$ & $\mathrm{RC} 5 \mathrm{~mm} \mathrm{MR} \mathrm{+RS} 7 \mathrm{~mm} \mathrm{LR}$ & $0^{*}$ \\
\hline 17 & 16 & $40 \mathrm{PD}$ & $\mathrm{RC} 5 \mathrm{~mm} \mathrm{MR} \mathrm{+RS} 8 \mathrm{~mm} \mathrm{LR}$ & $4 \mathrm{PD}$ \\
\hline 18 & 15 & $40 \mathrm{PD}$ & $\mathrm{RC} 5 \mathrm{~mm} \mathrm{MR}+\mathrm{RS} 8 \mathrm{~mm} \mathrm{LR}$ & $8 \mathrm{PD}$ \\
\hline 19 & 12 & $40 \mathrm{PD}$ & $\mathrm{RC} 5 \mathrm{~mm} \mathrm{MR}+\mathrm{RS} 8 \mathrm{~mm} \mathrm{LR}$ & $2 \mathrm{PD}$ \\
\hline 20 & 12 & $40 \mathrm{PD}$ & $\mathrm{RC} 5 \mathrm{~mm} \mathrm{MR} \mathrm{+RS} 8 \mathrm{~mm} \mathrm{LR}$ & $8 \mathrm{PD}$ \\
\hline 21 & 16 & $40 \mathrm{PD}$ & $\mathrm{RC} 5 \mathrm{~mm} \mathrm{MR}+\mathrm{RS} 8 \mathrm{~mm} \mathrm{LR}$ & $8 \mathrm{PD}$ \\
\hline 22 & 10 & $40 \mathrm{PD}$ & $\mathrm{RC} 5 \mathrm{~mm} \mathrm{MR}+\mathrm{RS} 8 \mathrm{~mm} \mathrm{LR}$ & $6 \mathrm{PD}$ \\
\hline 23 & 10 & $45 \mathrm{PD}$ & $\mathrm{RC} 6 \mathrm{~mm} \mathrm{MR} \mathrm{+RS} 7 \mathrm{~mm} \mathrm{LR}$ & $10 \mathrm{PD}$ \\
\hline 24 & 10 & $45 \mathrm{PD}$ & $\mathrm{RC} 6 \mathrm{~mm} \mathrm{MR}+\mathrm{RS} 8 \mathrm{~mm} \mathrm{LR}$ & $10 \mathrm{PD}$ \\
\hline 25 & 17 & $45 \mathrm{PD}$ & $\mathrm{RC} 6 \mathrm{~mm} \mathrm{MR} \mathrm{+RS} 8 \mathrm{~mm} \mathrm{LR}$ & $10 \mathrm{PD}$ \\
\hline 26 & 10 & 45 PD & $\mathrm{RC} 6 \mathrm{~mm} \mathrm{MR}+\mathrm{RS} 7 \mathrm{~mm} \mathrm{LR}$ & $10 \mathrm{PD}$ \\
\hline 27 & 15 & $45 \mathrm{PD}$ & $\mathrm{RC} 6 \mathrm{~mm} \mathrm{MR} \mathrm{+RS} 7 \mathrm{~mm} \mathrm{LR}$ & $8 \mathrm{PD}$ \\
\hline 28 & 10 & $45 \mathrm{PD}$ & $\mathrm{RC} 6 \mathrm{~mm} \mathrm{MR} \mathrm{+RS} 8 \mathrm{~mm} \mathrm{LR}$ & $10 \mathrm{PD}$ \\
\hline 29 & 10 & $45 \mathrm{PD}$ & $\mathrm{RC} 6 \mathrm{~mm} \mathrm{MR}+\mathrm{RS} 8 \mathrm{~mm} \mathrm{LR}$ & $10 \mathrm{PD}$ \\
\hline 30 & 15 & $45 \mathrm{PD}$ & $\mathrm{RC} 6 \mathrm{~mm} \mathrm{MR}+\mathrm{RS} 7 \mathrm{~mm} \mathrm{LR}$ & $10 \mathrm{PD}$ \\
\hline 31 & 12 & $45 \mathrm{PD}$ & $\mathrm{RC} 6 \mathrm{~mm} \mathrm{MR} \mathrm{+RS} 7 \mathrm{~mm} \mathrm{LR}$ & $8 \mathrm{PD}$ \\
\hline Mean & 12.80 & $36.93 \mathrm{PD}$ & & 4.48 PD \\
\hline
\end{tabular}

\begin{tabular}{|c|c|c|c|c|}
\hline Patient $\mathrm{N}^{\circ}$ & Age (years) & Preoperative deviation & Surgical planning & Postoperative deviation ( 3 months) \\
\hline 1 & 18 & $20 \mathrm{PD}$ & $\mathrm{RC} 5 \mathrm{~mm} \mathrm{LR}+\mathrm{RS} 4 \mathrm{~mm} \mathrm{MR}$ & $0^{*}$ \\
\hline 2 & 11 & $35 \mathrm{PD}$ & $\mathrm{RC} 6 \mathrm{~mm} \mathrm{LR}+\mathrm{RS} 5 \mathrm{~mm} \mathrm{MR}$ & $2 \mathrm{PD}$ \\
\hline 3 & 15 & $35 \mathrm{PD}$ & $\mathrm{RC} 6 \mathrm{~mm} \mathrm{LR}+\mathrm{RS} 5 \mathrm{~mm} \mathrm{MR}$ & $2 \mathrm{PD}$ \\
\hline 4 & 15 & $35 \mathrm{PD}$ & $\mathrm{RC} 6 \mathrm{~mm}$ LR +RS $5 \mathrm{~mm}$ MR & $2 \mathrm{PD}$ \\
\hline 5 & 15 & $35 \mathrm{PD}$ & $\mathrm{RC} 6 \mathrm{~mm} \mathrm{LR}+\mathrm{RS} 5 \mathrm{~mm} \mathrm{MR}$ & $4 \mathrm{PD}$ \\
\hline 6 & 13 & $35 \mathrm{PD}$ & $\mathrm{RC} 6 \mathrm{~mm} \mathrm{LR}+\mathrm{RS} 5 \mathrm{~mm} \mathrm{MR}$ & $0^{*}$ \\
\hline 7 & 11 & $35 \mathrm{PD}$ & $\mathrm{RC} 6 \mathrm{~mm}$ LR +RS $5 \mathrm{~mm}$ MR & $0^{*}$ \\
\hline 8 & 10 & $35 \mathrm{PD}$ & $\mathrm{RC} 6 \mathrm{~mm} \mathrm{LR}+\mathrm{RS} 5 \mathrm{~mm} \mathrm{MR}$ & $0^{*}$ \\
\hline 9 & 11 & $35 \mathrm{PD}$ & $\mathrm{RC} 6 \mathrm{~mm} \mathrm{LR}+\mathrm{RS} 5 \mathrm{~mm} \mathrm{MR}$ & $4 \mathrm{PD}$ \\
\hline 10 & 17 & $45 \mathrm{PD}$ & $\mathrm{RC} 6 \mathrm{~mm} \mathrm{LR}+\mathrm{RS} 8 \mathrm{~mm} \mathrm{MR}$ & 5 PD \\
\hline Mean & 13,6 & 34,5 PD & & 1,8 PD \\
\hline
\end{tabular}

a good outcome ${ }^{(9)}$. Careful inspection of the ocular globe after anesthesia is recommended because the diagnosis of perforation is made at the moment of anesthesia only in $50 \%$ of the cases and an injection of at least 0.3 to $0.5 \mathrm{ml}$ is necessary to achieve an increase of clinically evident intraocular pressure with corneal edema ${ }^{(10-11)}$.

Cheng et al. conducted a study comparing the morbidity of retrobulbar and general anesthesia in monocular strabis- 
mus surgery. In their study, there were no differences regarding postoperative nausea and vomiting associated with the two techniques. The group that received retrobulbar anesthesia experienced less immediate postoperative discomfort $(\mathrm{p}<0.01)$, greater levels of activity $(\mathrm{p}<0.0001)$ while in the hospital and was discharged from the hospital sooner $(\mathrm{p}<0.001)^{(12)}$. Another study evaluated the risk of myocardial ischemia in cataract surgery after peribulbar and general anesthesia and showed a significant lower risk of myocardial ischemia in peribulbar anesthesia than in general anesthesia in cataract surgery ${ }^{(13-14)}$. There are no studies on strabismus surgery comparing the risk of myocardial ischemia in peribulbar and general anesthesia.

Another study that compared strabismus surgery under propofol sedation with local versus general anesthesia showed that times from incision closure to leaving the operating room and to hospital discharge are decreased in local anesthesia compared with those of general anesthesia ${ }^{(15)}$.

For ethical reasons, general anesthesia was reserved for children and adolescents because the availability of this type of anesthesia is not sufficient for all patients. And peribulbar anesthesia could not be offered to children and young adolescents since collaboration of patients during local anesthesia is necessary. Due to this fact, patients in the group of general anesthesia were younger, but statistical analysis used for adjusting the age variable in the postoperative results showed no statistical difference.

According to postoperative results of this study we recommend to consider the use of peribulbar anesthesia in adult and collaborative patients undergoing strabismus surgery for small and moderate angles of deviation.

\section{RESUMO}

Objetivo: Comparar os resultados de cirurgias de estrabismo sob anestesia peribulbar e geral em casos de estrabismos horizontais de pequeno e médio ângulo. Métodos: Foram avaliados os prontuários médicos de 84 pacientes com estrabismo horizontal de pequeno e médio ângulo submetidos à cirurgia de estrabismo. Quarenta e dois pacientes foram submetidos à cirurgia sob anestesia peribulbar e quarenta e dois sob anestesia geral. A cirurgia foi considerada satisfatória quando o desvio pós-operatório era de até 10 dioptrias prismáticas (DP). Resultados: A cirurgia foi considerada satisfatória em todos os pacientes. O teste de Mann-Whitney não mostrou diferença no ângulo pré-operatório $(\mathrm{p}=0,366)$ nem nos resultados pósoperatórios $(\mathrm{p}=0,800)$ entre os dois grupos. Ajustando as variá- veis idade e tipo de estrabismo (esotropia e exotropia), o teste ANCOVA (análise de covariância) não mostrou diferença significativa entre os grupos $(\mathrm{p}=0,368)$. Não houve complicações decorrentes da cirurgia ou técnica anestésica em nenhum dos grupos. Conclusões: Este estudo sugere que não há diferença nos resultados pós-operatórios da cirurgia de estrabismo entre anestesia peribulbar e geral em estrabismos horizontais de pequeno e médio ângulo.

Descritores: Anestesia local/métodos; Estrabismo/cirurgia; Procedimentos cirúrgicos oftalmológicos; Transtornos da motilidade ocular

\section{REFERENCES}

1. Sanders JR, Nelson LB, Deutsch JA. Peribulbar anesthesia for strabismus surgery. Am J Ophthalmol. 1990;109(6):705-8.

2. Szmyd SM, Nelson LB, Calhoun JH, Harley RD. Retrobulbar anesthesia in strabismus surgery. Arch Ophthalmol. 1984;102(9):1325-7.

3. Yu CB, Wong VW, Fan DS, Yip WW, Lam DS. Comparison of lidocaine $2 \%$ gel versus amethocaine as the sole anesthetic agent for strabismus surgery. Ophthalmology. 2003;110(7):1426-9.

4. Davis DB 2 $2^{\text {nd }}$, Mandel MR. Efficacy and complication rate of 16,224 consecutive peribulbar blocks. A prospective multicenter study. J Cataract Refract Surg. 1994;20(3):327-37. Erratum in: J Cataract Refract Surg. 1994; 20(6):673. Comment in: J Cataract Refract Surg. 1994;20(6):673. J Cataract Refract Surg. 1995;21(1):4.

5. Wang HS. Peribulbar anesthesia for ophthalmic procedures. J Cataract Refract Surg. 1988;14(4):441-3.

6. Hamilton RC. Complications of ophthalmic regional anesthesia. Ophthalmol Clin North Am. 1998;11:99-114.

7. Prieto-Díaz J, Souza-Dias C. Cirurgia do estrabismo. In: Prieto-Díaz J, Souza-Dias C. Estrabismo. 4 $4^{\underline{a}}$ ed. São Paulo: Livraria Santos Editora; 2002. p.465-514.

8. Ripart J, Lefrant JY, de La Coussay JE, Prat-Pradal D, Vivien B, Eledjam JJ. Peribulbar versus retrobulbar anesthesia for ophthalmic surgery: an anatomical comparison of extraconal and intraconal injections. Anesthesiology. 2001; 94(1):56-62. Comment in: Anesthesiology. 2001;95(6):1533-5.

9. Holekamp NM, Wax MB. Intraocular anesthetic following peribulbar anesthesia. Arch Ophthalmol. 1998;116(3):380-1.

10. Feibel RM. Checking for globe perforation after retrobulbar injection. Surv Ophthalmol. 1995;40(1):86. Comment on: Surv Ophthalmol. 1995;39(4): 344-5.

11. Lavrich JB, Nelson LB. Local anaesthesia for strabismus surgery. Ophthalmol Clin North Am. 1992;5:131-41.

12. Cheng KP, Larson CE, Biglan AW, D'Antonio JA. A prospective, randomized controlled comparison of retrobulbar and general anesthesia for strabismus surgery. Ophthalmic Surg. 1992;23(9):585-90.

13. Glantz L, Drenger B, Gozal Y. Perioperative myocardial ischemia in cataract surgery patients: general versus local anesthesia. Anesth Analg. 2000;91(6): 1415-9. Comment in: Anesth Analg. 2001;93(2):518.

14. Cozal Y, Cohen J, Glantz L, Drenger B. Incidence of perioperative myocardial ischemia in cataract surgery: comparison of local and general anesthesia. Anesthesiology. 2001;94(2):376.

15. Greenberg MF, Pollard ZF. Adult strabismus surgery under propofol sedation with local versus general anesthesia. J AAPOS. 2003;7(2):116-20. 